\title{
Un « nouvel art de vivre ensemble » : quelles expérimentations sociales dans les quartiers dits durables ? L'exemple du quartier Beauregard à Rennes
}

\section{A "new art of living together" : which social experiments in sustainable} neighborhoods? The example of Beauregard district in Rennes

Ein «neues Modell des Zusammenlebens": welche sozialen Erprobungen in sogenannten nachhaltigen Stadtvierteln? Das Beispiel des «Beauregard" Viertels in Rennes

\section{François Valegeas}

\section{(Q) OpenEdition}

Electronic version

URL: http://journals.openedition.org/rge/5279

DOI: $10.4000 /$ rge.5279

ISSN: $2108-6478$

\section{Publisher}

Association des géographes de l'Est

Printed version

Date of publication: 30 December 2014

ISSN: 0035-3213

\section{Electronic reference}

François Valegeas, « Un « nouvel art de vivre ensemble » : quelles expérimentations sociales dans les quartiers dits durables ? L'exemple du quartier Beauregard à Rennes », Revue Géographique de l'Est [Online], vol. 54 / n³-4 | 2014, Online since 03 March 2015, connection on 08 September 2020. URL : http://journals.openedition.org/rge/5279; DOI : https://doi.org/10.4000/rge.5279 


\title{
Un « nouvel art de vivre ensemble » : quelles expérimentations sociales dans les quartiers dits durables? L'exemple du quartier Beauregard à Rennes
}

\author{
A "new art of living together" : which social experiments in sustainable \\ neighborhoods? The example of Beauregard district in Rennes \\ Ein "neues Modell des Zusammenlebens": welche sozialen Erprobungen in \\ sogenannten nachhaltigen Stadtvierteln? Das Beispiel des «Beauregard" \\ Viertels in Rennes
}

François Valegeas

\section{Un « nouvel art de vivre ensemble » : quelles expérimentations sociales dans les quartiers dits durables ? L'exemple du quartier Beauregard à Rennes}

L'action sur la ville est aujourd'hui placée sous le signe d'un développement urbain durable, cherchant à apporter une réponse aux problématiques des villes contemporaines. En effet, celles-ci seraient le terrain privilégié de mise en œuvre d'un développement durable car elles concentreraient les impacts des crises environnementales et sociales, mais aussi en raison des potentialités qu'elles offrent. C'est dans cet esprit qu'ont émergé en Europe des projets de quartiers dits « durables » ${ }^{1}$, qui permettent, par la territorialisation de la mise en œuvre de ce développement durable, de le rendre concret et appropriable (Theys, 2002). Ces projets ont l'ambition de créer des quartiers innovants, avec un impact environnemental faible et apportant une haute qualité de vie à leurs habitants. De tels projets se multiplient depuis une 
quinzaine d'années en France, souvent dans le cadre d'appels à projets ou de labels qui tentent de structurer ces initiatives et de faire émerger des référentiels communs.

Les questions soulevées ont pour ambition de réinterroger nos modes de faire et de penser les villes en vue d'engager un changement des pratiques. Mais des divergences apparaissent: si l'idée de changement est centrale, la définition et les projets de transformation qui sous-tendent ce développement urbain durable ne sont guère explicités. Pour les chercheurs analysant ses effets sur les politiques urbaines, différentes approches peuvent être discernées : le développement durable est perçu par certains comme un transformateur des politiques locales (Hamman, 2012), voire un référentiel en devenir de l'action publique urbaine (Ascher, 2004). D'autres chercheurs critiquent son éloquence improductive (Lascoumes, 2001), ou encore l'utilisation d'un «concept creux» (Boutaud, 2004) qui justifierait des politiques urbaines toujours aussi injustes socialement et destructrices écologiquement (Béal, 2011). En fait, ce développement urbain durable s'apparente davantage à un mot d'ordre mobilisateur pour l'action qu'un projet politique stabilisé.

Ces quartiers dits durables sont de bons révélateurs des potentialités et contradictions de ce développement urbain durable. Ne sont-ils que des espaces-témoins de la mise en œuvre d'éco-techniques à visée performative? Ou peuvent-ils être des lieux de construction de projets collectifs, des vecteurs de changement dans la fabrique de la ville?

Dans cet article nous focaliserons notre regard sur la manière dont les appels à projets envisagent le rôle des habitants dans ces quartiers. Ils sont les révélateurs d'une normalisation des réponses aux enjeux sociaux. D'autre part, à partir d'une recherche menée dans un quartier considéré comme précurseur en France, nous analyserons les formes d'engagement des habitants, l'émergence de dynamiques collectives, véritables potentialités pour ce changement attendu.

5 Cet article s'appuie sur une recherche de thèse en cours. Nous analysons la construction d'un « référentiel » des quartiers dits durables par différentes démarches cherchant à développer de tels projets (appels à projets, guides de "bonnes pratiques ", benchmarks), mis en regard avec l'analyse d'opérations en cours (une quinzaine dans toute la France).

6 Un travail de terrain vient compléter cette recherche, afin de comprendre les formes d'appropriations qui se construisent dans certains quartiers dont Beauregard à Rennes est un exemple intéressant. Dans ce quartier, nous avons mené 32 entretiens semidirectifs avec des habitants entre 2011 et 2013, complétés par le suivi d'une démarche participative (réalisation de parcours commentés, murs de paroles, ateliers participatifs...) menée par les acteurs locaux : associations, aménageur, ville.

\section{Quartiers dits durables et réponses aux enjeux sociaux}

7 Les quartiers dits durables sont issus d'un héritage double, qui est révélateur de conceptions assez différentes de ce que serait un développement urbain durable et de la manière dont les habitants sont amenés à s'impliquer dans les projets qui s'en réclament. 
Des auteurs mettent ainsi en avant les expériences écologistes des années 1970 comme l'une des filiations des quartiers durables actuels ${ }^{2}$. Organisés sur un mode communautaire, ces quartiers ont été pensés avant tout comme des espaces de contestation, dans lesquels s'inventèrent des alternatives collectives basées sur une écologie parfois radicale.

9 Les quartiers dits durables actuels puisent bien souvent leurs principes dans la mise en œuvre d'un développement durable plus normalisé, tel qu'il a été formulé dans le rapport Brundtland de 1987. Ces quartiers sont généralement des opérations menées par des collectivités locales, d'abord comme vitrines des avancées éco-techniques locales puis à partir des années 1990 en devenant des «quartiers-types » (Souami, 2009, p.33), davantage ancrés dans les modes de décision, de financement et de construction traditionnels. Dans ces quartiers tels qu'ils se sont répandus en Europe, la place des habitants et de leurs initiatives est bien souvent restreinte, et relève d'un rôle d'usagers que leur ont assigné les concepteurs des projets.

10 En France, le ministère en charge du développement durable fait le constat au cours des années 2000 d'un retard sur de telles démarches à l'échelle du quartier et fixe l'objectif lors du Grenelle de l'environnement d'« au moins un ÉcoQuartier avant 2012 [...] dans toutes les communes qui ont des programmes de développement de l'habitat significatif $»^{3}$. Il s'agit pour l'État d'impulser une dynamique nationale en encourageant les collectivités locales à s'engager dans la réalisation de projets locaux, par le lancement de plusieurs appels à projets ${ }^{4}$. La démarche ÉcoQuartier prend la forme d'un concours national afin de primer les projets les plus exemplaires, et propose un accompagnement des collectivités candidates. En cela, les guides, grilles d'évaluations, documents pédagogiques nous permettent de mieux saisir les principes et présupposés de ce référentiel en cours de formalisation.

\section{A. Le « vivre ensemble » comme mot d'ordre}

11 Ces appels à projets se basent sur des principes constituant un cadre en cours de formalisation pour la fabrique de ces quartiers. L'analyse des grilles d'évaluation révèle une volonté de s'inspirer des modèles européens tout en singularisant un «modèle français " prenant davantage en charge les enjeux sociaux. Cherchant à se différencier de projets critiqués pour être devenus des " ghettos verts ", des quartiers participant à une ségrégation socio-spatiale, les appels à projets incitent les collectivités à «traduire dans le projet la mixité et la diversité sociales, urbaines et fonctionnelles dans le quartier " (MEDDL, 2008).

12 La succession des appels à projets révèle une évolution de la prise en charge des enjeux sociaux dans la mise en œuvre d'un développement durable.

13 Si dès la première grille de 2008, l'objectif est de développer « un nouvel art de vivre ensemble dont l'ÉcoQuartier est en passe de devenir le symbole » (MEDDL, 2008), les outils se révèlent assez traditionnels et sectoriels. Les dimensions sociales apparaissent comme une garantie contre la ségrégation pour des projets d'abord techniques, et véhiculent des discours relativement normatifs et fortement décontextualisés. Plusieurs chercheurs pointent ainsi le risque de répétition des erreurs du passé par la construction de modèles urbains décontextualisés : « on pourrait être enclin à se demander si les vertus prêtées aux éco-quartiers ne sont pas en train de nous conduire aux mêmes impasses 
que les belles espérances des villes nouvelles et des grands ensembles " (Bonard, Matthey, 2010).

14 Les enjeux sociaux ne sont pas réellement retravaillés par la durabilité. La mise en œuvre de ce "vivre ensemble» reste largement restreinte à la recherche d'une diversité des populations, pour à la fois éviter une sélectivité sociale de ces quartiers, mais aussi « recréer ou [...] renforcer le lien entre les êtres humains, sociétés et territoires afin de favoriser le «mieux vivre» ensemble» (MEDDTL, 2011, p.25). La mixité sociale serait alors un élément majeur de cette cohésion sociale que cherche à favoriser le développement durable. Sa mise en œuvre permettrait ainsi tout à la fois d'équilibrer le peuplement dans les territoires, de diversifier l'offre de logements, de créer du lien social ou encore d'intégrer les populations dans la ville: "une potion magique qui doit résoudre tout à la fois la question des inégalités et la question du désordre social » (Lelévrier, 2006). Pour réaliser cette diversité du peuplement, les décideurs s'appuient sur la programmation résidentielle, en jouant sur les méthodes de financement, les statuts et les tailles des logements.

De même, la question de l'implication habitante est souvent réduite à la promotion de dispositifs de participation institutionnelle, portant sur des objets précis (d'abord autour de la "vie de quartier", parfois sur certains espaces publics), et dont les objectifs relèvent avant tout d'une acceptation du projet et de ses principes, et d'une pédagogie autour des pratiques à adopter. Au-delà de quelques exemples d'une volonté d'implication des habitants dans la conception des projets, la majorité des projets considère les habitants comme des individus passifs, à responsabiliser.

16 La nouvelle grille parue en 2010, évolue sensiblement sur les dimensions sociales. Il s'agit là d'engager une réflexion sur les modes de vie, que cela soit dans l'adoption d'éco-gestes ou dans l'adaptation des usages aux exigences des éco-techniques mises en place. Les premiers retours d'expériences ont en effet montré des « effets-rebonds » et des usages mal adaptés qui ne permettaient pas d'atteindre les performances recherchées. Les objectifs fixés ne pourraient alors être atteints que par un changement des pratiques des habitants. Comme l'explique le coordinateur de la démarche au ministère, «le premier appel à projet était vraiment un test [...]. Depuis, on a beaucoup réfléchi sur les résultats et on a mis l'accent sur le côté usages des projets [...]. Ce n'est pas le quartier qui est durable, ce sont les usages des habitants $"^{5}$. L'appel à projets incite à prendre en compte la gestion des comportements des futurs habitants. Malgré tout, les enjeux d'appropriation de ces quartiers et des projets collectifs qui y seraient liés ne sont pas abordés.

17 Finalement, ce « vivre ensemble » proposé comme projet social pour ces quartiers nous semble davantage un slogan qu'un véritable mode de renouvellement des réflexions et pratiques. Il permet d'inclure des préoccupations variées dans un mot-valise, comme l'explicite Guy Di Méo, "le "vivre ensemble» ne se définit pas aisément et souffre (ou bénéficie?) d'acceptions multiples: un peu à la carte, en quelque sorte... ${ }^{6}$. Mais, plus contradictoire avec les ambitions affichées, il entérine une démarche basée sur des présupposés peu questionnés (la mixité sociale, la cohésion sociale) et appelant à la responsabilisation individuelle, sans proposer de cadre à l'appréhension des phénomènes structurels, à la prise en compte des formes de domination ou aux processus d'émancipation qui pourraient voir le jour. 


\section{B. Analyser la construction de dynamiques collectives locales}

L'appel à ce «nouvel art de vivre ensemble» (MEDDL, 2008) nous semble néanmoins intéressant en ce qu'il propose ou implique en termes de dynamiques collectives dans ces quartiers. L'analyse de la manière dont les quartiers dits durables envisagent l'implication des habitants, l'étude du quartier comme espace social support de ce "vivre ensemble», nous semblent des questionnements riches pour comprendre les logiques qui sous-tendent la construction de tels projets.

\section{Le quartier dit durable : opération technique locale ou espace d'expérimentation sociale?}

En France, les projets sont marqués par des "logiques descendantes et techniques " (Renauld, 2012) à visée performative. Les retours d'expériences ont montré que les décalages observés entre projets et performances réelles sont liées à des comportements inadéquats des habitants. Les habitants sont ainsi perçus sous deux angles dans les projets (Zetlaoui-Léger et al., 2013). Ils sont tout d'abord pointés comme des habitants à "responsabiliser», en les sensibilisant aux éco-techniques et en les éduquant aux « bonnes pratiques » à adopter. Ils ont là un rôle passif puisqu'il s'agit de faire changer leurs habitudes, selon une grille de valeurs hiérarchisées par des « experts». Divers outils permettent ainsi de rapprocher ces modes de vie de la figure idéalisée de l' "éco-habitant »: des aménagements spécifiques contraignant les usages (qu'ils soient par exemple des «dispositifs par omission" (Boisonnade, 2011) ou des " nudges verts» (Conseil d'Analyse Stratégique, 2011)), ou encore des guides de bonnes pratiques à destination des habitants.

D'autre part, les habitants sont considérés comme des futurs « gestionnaires » : l'accent est mis sur le rôle que les habitants joueront dans le fonctionnement du futur quartier. Il y a donc une certaine exigence auprès de ces habitants pour qu'ils prennent en charge la gestion quotidienne des dispositifs et la vie sociale du quartier (Souami, 2009).

Nous y voyons un double enjeu qui se joue à la fois dans les relations entre les écotechniques et leurs usages, et entre les changements individuels et collectifs : l'objectif est-il de pousser à un changement des modes de vie individuels pour les rendre compatibles avec les technologies employées, en escomptant que la somme de ces changements améliorera l'empreinte écologique globale ? L'acceptabilité des projets est donc là un enjeu majeur pour la normalisation des comportements. Ou la question estelle plus globalement de réinventer des espaces de vie collective, des normes collectives partagées et appropriées ? L'entrée prend alors un sens politique ${ }^{7}$, la technique venant servir un intérêt commun redéfini à l'aune des enjeux contemporains. Cet impensé des projets interroge sur leur capacité à dépasser une approche technique pour prendre en compte l'enjeu de la construction d'un devenir collectif.

Dans le cadre de notre recherche, il nous semble intéressant de comprendre comment des modes d'habiter individuels ou collectifs peuvent s'y construire. Or il s'agit d'un angle mort de la recherche, alors même que selon certains auteurs, les modes de vie, d'habiter et d'engagements seraient des ressources premières sur lesquelles la durabilité urbaine devrait s'appuyer (Raineau, 2009 ; Theys et al. 2010). 


\section{Le quartier, une échelle de l'engagement?}

Si les références à l'échelle du quartier sont omniprésentes, les potentialités et limites de cette échelle restent peu analysées comme telles dans le cas des quartiers durables. Dans les appels à projets, le quartier apparaît moins comme un espace de constructions collectives que comme une échelle d'action, un « terrain qui semble pertinent pour mettre à l'épreuve les processus de développement local, où convergent les préoccupations de gestion publique des élus municipaux et la demande de qualité du cadre de vie quotidien, de la part des habitants» (PUCA, 2006). Pour certains, l'échelle même de ces projets, aux vertus intégratrices, favoriserait un "vivre-ensemble", permettrait d'impulser une vie de quartier et de remédier à une perte constatée du lien social. Mais peu de choses sont dites sur les moyens de favoriser ce "vivre-ensemble ", comme si la seule évocation du quartier suffisait à rendre naturelle une certaine cohésion sociale.

À la suite de nombreux auteurs, nous faisons l'hypothèse que le quartier reste une référence pertinente dans la compréhension des rapports des habitants à leur territoire (Lefebvre, 1967 ; Morin, Rochefort, 1998 ; Authier et al. 2006). Il constitue une échelle significative dans l'établissement du lien social, d'une part par l'enracinement de pratiques individuelles et collectives, mais aussi parce qu'il peut devenir l'objet d'attachements particuliers par des habitants. Le quartier est un espace où se crée du collectif et l'affirmation de distinctions, et peut devenir un lieu d'expérimentations et d'initiatives collectives. Il pourrait alors être un pivot pour l'engagement des habitants, prenant autant la forme d'un rapport sensible avec cet espace et ce qui le compose (aménités, environnement, symbolique) ou d'une implication dans la construction collective d'un devenir commun.

\section{Le quartier durable comme moteur pour l'engagement local : l'exemple de Beauregard}

Dans la seconde partie de cet article, nous analyserons les dynamiques autour d'un projet de quartier dit durable, afin de mettre à jour les liens qui peuvent exister entre un projet d'abord éco-technique et l'émergence d'un projet collectif local. Si le cas de Beauregard à Rennes s'avère antérieur au développement des quartiers dits durables en France, les principes qui ont guidé sa conception en sont assez proches ${ }^{8}$. Néanmoins, l'antériorité de son peuplement par rapport à d'autres exemples français et les dynamiques collectives qui s'y déroulent permettent de mettre en exergue les potentialités d'une réflexion sur les processus d'appropriations voire d'engagements locaux.

26 Le projet de Beauregard est l'un des derniers projets urbains menés au cœur de Rennes. Programmé sur d'anciens terrains agricoles au Nord-Ouest de la ville, à proximité du grand ensemble de Villejean (et de son université), le quartier de Beauregard constitue l'une des dernières opportunités foncières intra-rocade. Le site étant présenté dès le schéma directeur de 1958 comme secteur de développement stratégique, des réserves foncières ont été créées par la ville dès les années 1980, ainsi qu'un pré-verdissement du quartier et un renforcement du bocage à partir de 1982. Le projet a démarré en 1995, avec plus de 3500 logements sortis de terre en 2013 (sur un total de 6000 prévus en 2022). Cette programmation sur le long terme (plus de 25 ans) est l'une des caractéristiques de l'opération, permettant des marges d'adaptation aux contraintes et 
à la demande sociale, comme nous le verrons par la suite. Il est programmé en trois zones d'aménagement concerté constituant le futur quartier de Beauregard. Nous traiterons ici surtout la zone centrale (la ZAC de Beauregard), constituant le cœur du quartier (comportant les équipements principaux, le parc, la place commerçante) et qui est beaucoup plus avancée que les deux autres.

\section{A. Un laboratoire du développement durable}

Beauregard est considéré comme un "laboratoire local ${ }^{9}$ du développement durable par les acteurs locaux : il s'agit en effet d'appliquer localement les outils stratégiques de l'agglomération tout en apportant des innovations supplémentaires. Comme le met en avant l'élu de quartier ${ }^{10}$, «le quartier Beauregard est le symbole des idéaux socialistes en matière d'aménagement urbain: accueil de tout type de population dans le respect d'un développement durable».

Ce quartier peut être considéré comme précurseur par les expérimentations écologiques qui y ont été menées: réseau de chaleur urbain, architecture bioclimatique, chartes de construction écologique... Des technologies alternatives de gestion de ces espaces ont été mises en œuvre à Beauregard, parfois ensuite reprises ailleurs: gestion différenciée des espaces verts, interdiction des produits phytosanitaires, etc. De plus, en 1995, les décideurs ont lancé une analyse environnementale du projet, expérimentale à l'époque, afin d'améliorer la qualité de vie future du quartier. Si les innovations techniques sont nombreuses, les porteurs du projet n'ont jamais utilisé directement ces dénominations de quartier durable ou d'écoquartier, à la fois car le projet a été défini avant le développement de ces réflexions en France, mais aussi car il apparaît en partie dépassé par les normes actuelles de construction durable: certaines innovations de l'époque s'étant généralisées voire étant rattrapées par la réglementation (notamment pour les objectifs de performance thermique).

Le projet de Beauregard cherche aussi à répondre à des enjeux sociaux par divers moyens. Pour l'architecte-urbaniste du projet, l'objectif est d'offrir un cadre de vie agréable, en apportant les bénéfices de la campagne dans la ville. Il s'agit alors de créer un "quartier vert » ou un "quartier paysager ", «composé à partir du concept paysager intimiste de la cité-jardin»: "nous souhaitions faire le bonheur des gens en leur donnant beaucoup de végétal et une qualité de vie résidentielle » (Ville de Rennes, 2007). La présence de la nature est renforcée par deux partis pris urbanistiques : la préservation des haies bocagères qui deviennent une trame pour le projet; et la réalisation d'« espaces verts du XXIème siècle $»^{11}$, ouverts sur le quartier. 
Figure 1 : Plan des espaces verts du quartier Beauregard

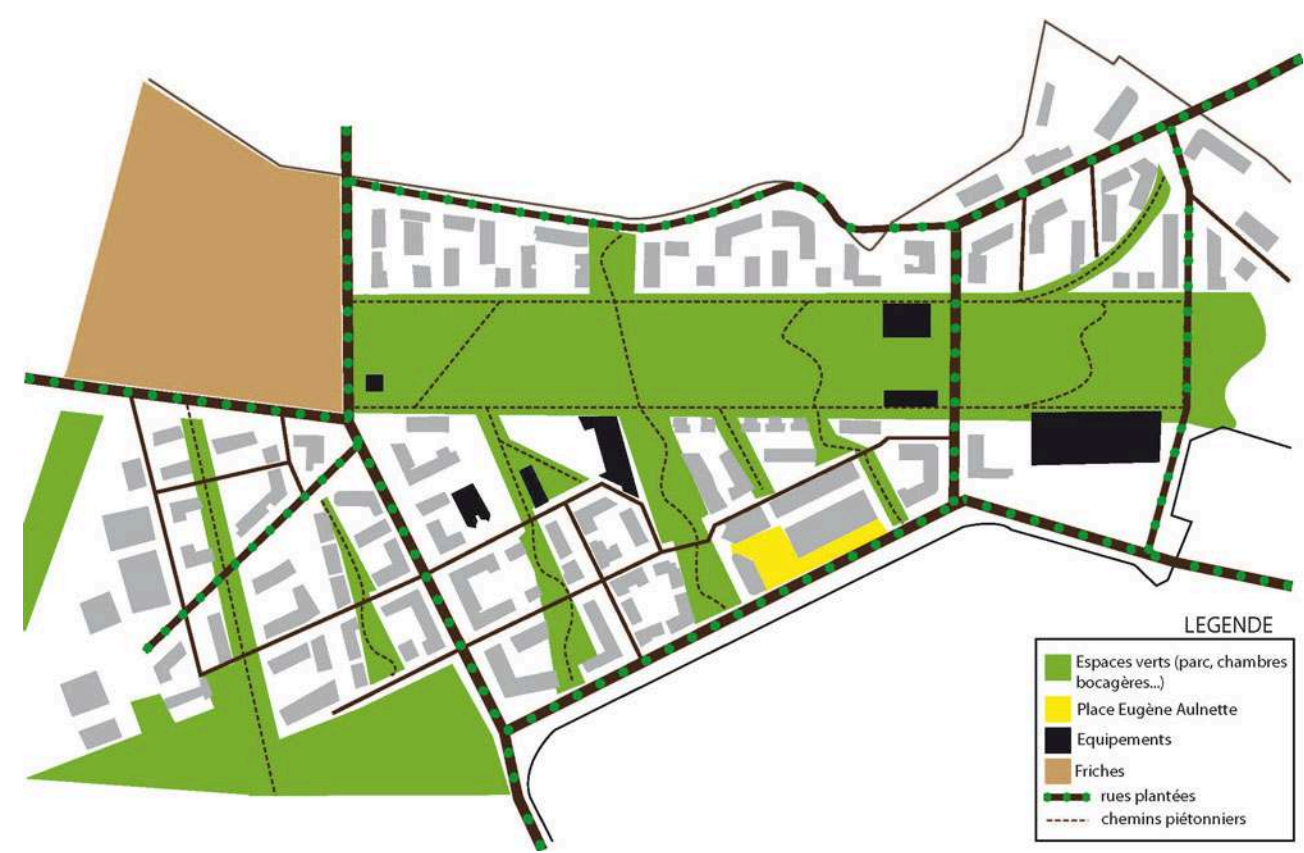

F. Valegeas, d'après Territoires et développement/Ville de Rennes, 2006

D'autre part, le quartier de Beauregard, qui s'inscrit pleinement dans la stratégie de l'agglomération, cherche à promouvoir une mixité sociale par des programmes de logements diversifiés. Le Programme Local de l'Habitat a pour objectif $d$ ' «offrir une gamme diversifiée de logements correspondant à la diversité des ménages" (PLH de Rennes, 2005). Les règles que l'agglomération s'est fixée sont ambitieuses : $25 \%$ de logements sociaux, $25 \%$ de logements intermédiaires et $50 \%$ de logements privés. Le projet de Beauregard cherche aussi à proposer une alternative de logement innovante pour de jeunes ménages avec enfants cherchant à accéder à la propriété, souvent contraints financièrement de se loger dans le périurbain. Sont alors mis en valeur la présence forte des espaces verts, mais aussi des formes urbaines et architecturales jugées attractives (comportant notamment des espaces extérieurs, réunis autour de jardins paysagers en cœur d'îlot).

Si la réponse aux enjeux sociaux passe d'abord par la recherche d'une diversité de l'habitat, les espaces collectifs sont mis en avant comme le moyen de réaliser une cohésion sociale dans ces quartiers. C'est cette vision qui est en partie reprise par certains élus : «Bien évidemment, un quartier ne se limite pas à ses logements. Une fois que les habitants ont pris possession de leurs biens, il s'agit pour les élus de créer les conditions de la construction d'une identité. Cela passe notamment par la construction de lieux et d'infrastructures permettant au lien social de se créer, de se conforter $»^{12}$. Le projet de Beauregard propose ainsi une diversité d'espaces, à la fois dans leur forme, mais aussi dans leurs usages possibles. Des espaces de proximité sont intégrés au projet afin de « créer une vie de quartier et de renforcer la cohésion sociale $»^{13}$. 
Figures 2 : Vue du quartier de Beauregard

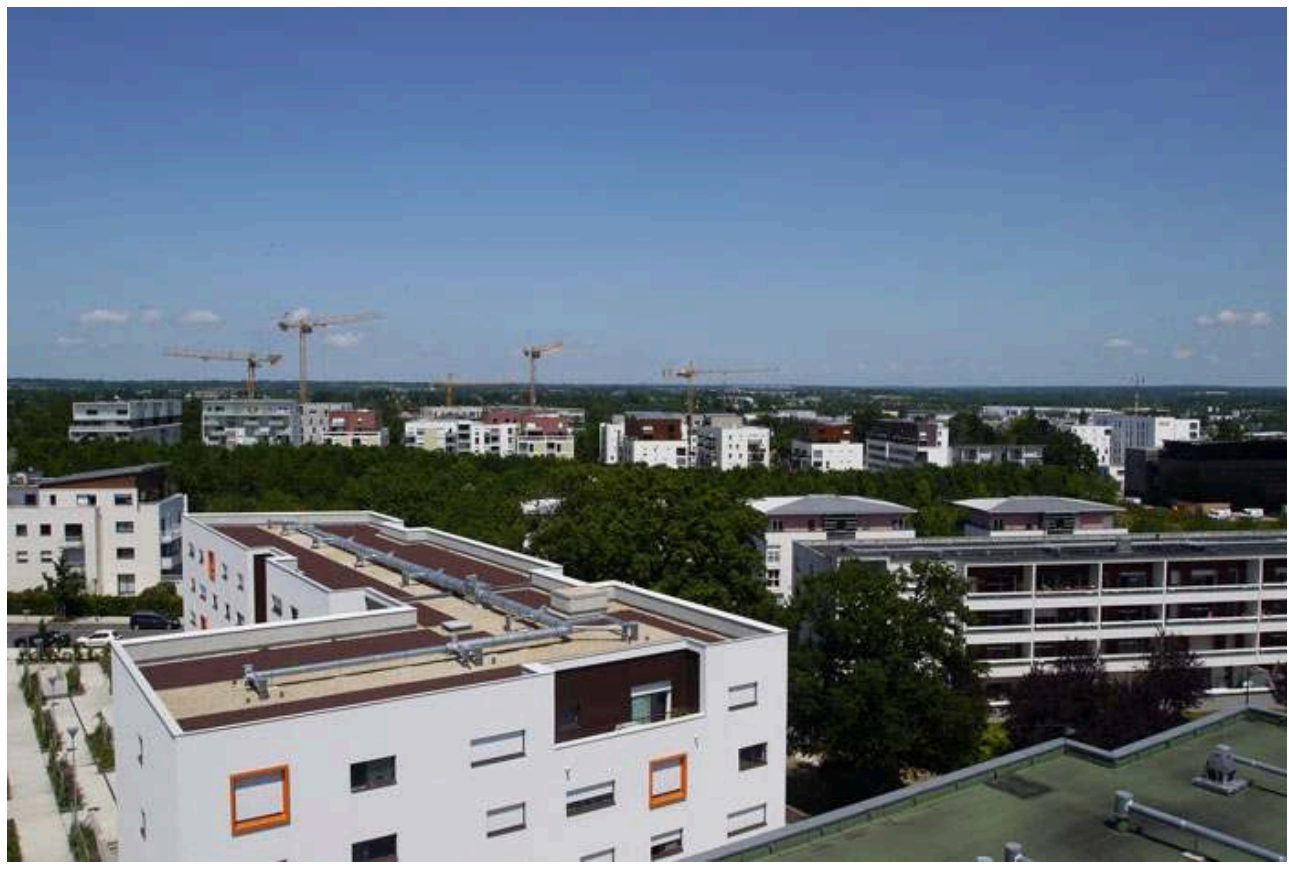

F. Valegeas, 2012

32 Des espaces publics de proximité forment la trame urbaine du quartier, et notamment une place bordée de commerces, et un parc central. L'objectif est ainsi « de donner une centralité au quartier, de créer un endroit où les gens vont, où ils se rencontrent. [...] En fait, on veut créer des occasions de vivre en dehors de son logement $»^{14}$.

La présence des espaces verts est supposée faciliter l'établissement de relations sociales. Leur relative ouverture et leur variété sont envisagées comme des atouts pour favoriser une diversité d'usages. La proximité de la nature est aussi perçue comme une manière de renouer un rapport sensible entre les habitants et leur environnement, de créer un lien au territoire afin de les inciter à s'approprier leur quartier. Pour l'association de quartier de Beauregard, qui a le projet de réaménager une zone humide en prolongement du parc, c'est «aussi un moyen d'impliquer les gens. On va mettre des panneaux pédagogiques sur les espèces d'arbres, d'oiseaux et tout. On va planter les arbres avec les enfants de l'école, en espérant qu'ils disent à leurs parents "vous voyez, c'est important ne pas faire n'importe quoi dans les zones humides" $»^{15}$. Ces espaces verts auraient donc à la fois une vocation paysagère et biologique mais aussi une utilité sociale et participeraient à la sensibilisation environnementale des habitants.

\section{B. Habiter à Beauregard : la constitution d'une communauté de destins}

34 Nos entretiens auprès des habitants montrent que le vécu de situations communes depuis les premières livraisons de logements, a permis l'émergence d'un sentiment d'appartenance à une communauté de destin. Plusieurs situations communes vécues par les habitants du quartier permettent d'expliquer l'émergence d'un « nous » qui leur est propre. 


\section{Vivre dans un quartier en construction : une mise à l'épreuve des premiers habitants}

Depuis 1998, les habitants sont amenés à côtoyer des chantiers en cours, et ses nuisances. Ces difficultés se sont doublées les premières années d'un manque d'équipements et de transports, ainsi que de l'enclavement relatif du quartier.

Les habitants rencontrés qui ont vécu ces premières années dans le quartier racontent les difficultés qu'ils ont pu connaître «les logements étaient à peine terminés, la peinture pas sèche [...] et puis le quartier était en plein chantier, il y avait de la poussière partout, des camions, des grues... $»^{16}$. Ce sentiment d'essuyer les plâtres a été une déception chez certains, le quartier n'étant pas celui qu'on leur avait promis lors de la vente : « on nous avait parlé d'un beau quartier, avec des belles images sur le papier. Mais on avait pas vraiment anticipé qu'il y aurait des travaux partout pendant quelques années ${ }^{17}$. D'autre part, de nombreux articles de journaux relatent l'absence de vie sociale et d'équipements pendant les premières années, Beauregard étant alors qualifié de " quartier-dortoir $»^{18}$.

Ces difficultés partagées par les premiers arrivants se sont notamment manifestées au cours des moments collectifs (premières réunions publiques notamment), lors desquels a émergé un sentiment de partage d'intérêts communs qui marque toujours les habitants qui sont restés : « il y avait une vraie solidarité. C'était pas facile tous les jours, mais au fur et à mesure qu'on discutait [...], on s'est rendu compte qu'on avait les mêmes difficultés, que c'était pas uniquement chacun de son côté $»^{19}$. Ce sentiment d'avoir été des pionniers dans le quartier est ainsi ambivalent : entre souvenir des difficultés vécues et nostalgie d'une solidarité forte.

\section{Des situations sociales et familiales proches qui fédèrent}

Malgré la diversité de l'habitat réelle dans le projet, le peuplement s'avère relativement homogène, par une sur-représentation importante des jeunes couples salariés avec un ou deux enfants, et des ménages à trajectoires résidentielles et sociales ascendantes ${ }^{20}$. Pour la grande majorité des ménages enquêtés, cette évolution résidentielle est associée à des bouleversements professionnels (premier emploi stable, mutation notamment depuis la région parisienne...) et familiaux (arrivée du premier enfant).

Cette homogénéité des situations sociales et familiales s'explique notamment par l'offre abondante dans le quartier de logements en accession, aidée ou non, vers lesquels les agences immobilières orientent bien souvent les potentiels acheteurs. La ville attirant beaucoup de jeunes ménages diplômés, ils trouvent à Beauregard des opportunités d'accéder à la propriété pour des biens récents, comportant des espaces extérieurs et des facilités de transport.

Cette mixité sociale plus relative que prévu fait l'objet d'un discours ambigu des élus locaux : «ce quartier est plutôt classes moyennes. Mais ce n'est pas grave, tant que ça vit bien. On ne peut pas obliger les gens à habiter là où ils ne veulent pas $»^{21}$. Et en effet, cette relative homogénéité du peuplement, que cela soit en termes de catégories sociales ou de compositions familiales, a conduit ici d'une part à la construction d'une représentation du quartier (" un quartier jeune, dynamique », " un quartier de classes moyennes »), mais aussi à un partage de préoccupations par les habitants eux-mêmes. 


\section{Des aspirations similaires : Beauregard, un «bon compromis »}

41 En effet, les ménages enquêtés partagent des aspirations communes et des modes de vie relativement similaires. Venir habiter Beauregard est d'abord l'expression d'une promotion sociale et résidentielle, de changements professionnels et familiaux, comme nous l'avons montré précédemment. Le déménagement est alors un moyen pour ces jeunes ménages d'arbitrer entre leurs moyens et leurs aspirations. Souvent, l'installation à Beauregard relève d'une stratégie bien déterminée, en privilégiant un logement et un cadre de vie de qualité, quitte à s'éloigner du centre-ville. Pour certains ménages, l'installation à Beauregard n'est pensée que comme une étape dans leur parcours résidentiel (avant l'installation en pavillon notamment). Le quartier de Beauregard apparait alors comme un « compromis acceptable ». La grande majorité des enquêtés présente le cadre de vie comme l'un des éléments majeurs expliquant leur installation dans le quartier, notamment dans la perspective de l'épanouissement de leurs enfants. Si l'habitat collectif n'est pas présenté comme un choix pour les ménages rencontrés, les aménités présentes dans le quartier et la situation par rapport au centre-ville et aux transports permettent de compenser cette contrainte.

Nos enquêtés apprécient les relations sociales et le calme du quartier, certains assimilant même Beauregard à un "village ", familial et convivial, en opposition à la figure des grandes villes. Un habitant explique ainsi : «c'est comme un village, c'est sympa, les gens se connaissent. C'est ce que je recherchais, j'en pouvais plus de Paris, où tout est gris, triste, les gens ne se parlent pas ». Ces discours rejoignent une représentation du village comme "figure spatialisée d'un vivre ensemble idéal », s'opposant à «la ville comme lieu de l'anonymat, de la solitude et du conflit » (Baudin, Dupuy, 2001).

\section{De l'habiter à l'engagement local}

43 Cette émergence d'une communauté de destins, appuyée sur des intérêts, des aspirations ou des modes de vie communs, s'est concrétisée par des formes d'engagement bien particulières à l'échelle du quartier.

\section{L'arrivée à Beauregard, une occasion de renégocier son engagement local}

M. Rautenberg et B. Lefebvre insistent sur l'« importance épistémologique de la rupture sociale et spatiale que représente le déménagement pour comprendre comment les vies se réajustent à un nouvel environnement" (2011). Déménager à Beauregard, c'est ainsi l'occasion de faire un bilan du passé, de mettre à l'épreuve ses aspirations résidentielles, de renégocier ses relations sociales, puis de s'approprier un quartier. Il s'agit d'opérer un "art de l'ajustement» (Ledrut, 1968) au sein duquel l'engagement personnel dans le quartier est remis en question.

En effet, l'arrivée à Beauregard concrétise un projet de vie nouveau pour les habitants, pour profiter de meilleures conditions de logement et d'un environnement agréable. Les habitants interrogés sont souvent enthousiastes quant à ce projet de quartier qui reste à imaginer, à construire. La plupart suit de près les chantiers en cours, par exemple lors de promenades régulières, voire comme quelques ménages en se constituant une revue de presse sur l'avancée du quartier. Cette construction du quartier, à la fois physique et symbolique, la plupart des habitants souhaite y prendre part, que cela soit en assistant aux réunions publiques ou plus encore, comme l'exprime 
cet habitant arrivé il y a un an et engagé au sein de l'association d'habitants: «le quartier ne se fera pas sans nous. Ici c'est un quartier tout neuf, c'est l'occasion de faire quelque chose de bien. [...] Il faut que chacun mette du sien pour qu'on y vive bien ${ }^{22}$. L'arrivée dans un quartier en train de se faire est souvent l'occasion de s'investir dans un défi collectif.

\section{Une cristallisation des attentes au sein de l'association d'habitants}

Face aux difficultés rencontrées, un groupe constitué d'habitants " pionniers » a lancé une mobilisation autour de l'implantation d'une crèche dans le quartier, obtenue après quelques mois de négociation et finalement livrée en $2008^{23}$. Le rôle de ces " pionniers" mobilisés, une fois constitués en association, leur a permis d'acquérir une légitimité tant auprès des autres habitants du quartier que des acteurs locaux. Cette volonté de participer pleinement à la création du quartier fonde le projet de l'association d'habitants Vivre à Beauregard créée en 2008 : ses statuts insistent sur la volonté de " prendre part à l'aménagement du quartier » et à "encourager le lien social ». Elle comporte aujourd'hui plus de 250 adhérents, qui participent de manière très diverse aux activités proposées, qu'elles soient culturelles, sportives, ou davantage liées à l'enfance ou au devenir du quartier.

Figure 3 : Vivre à Beauregard, organisateur de la fête annuelle de quartier

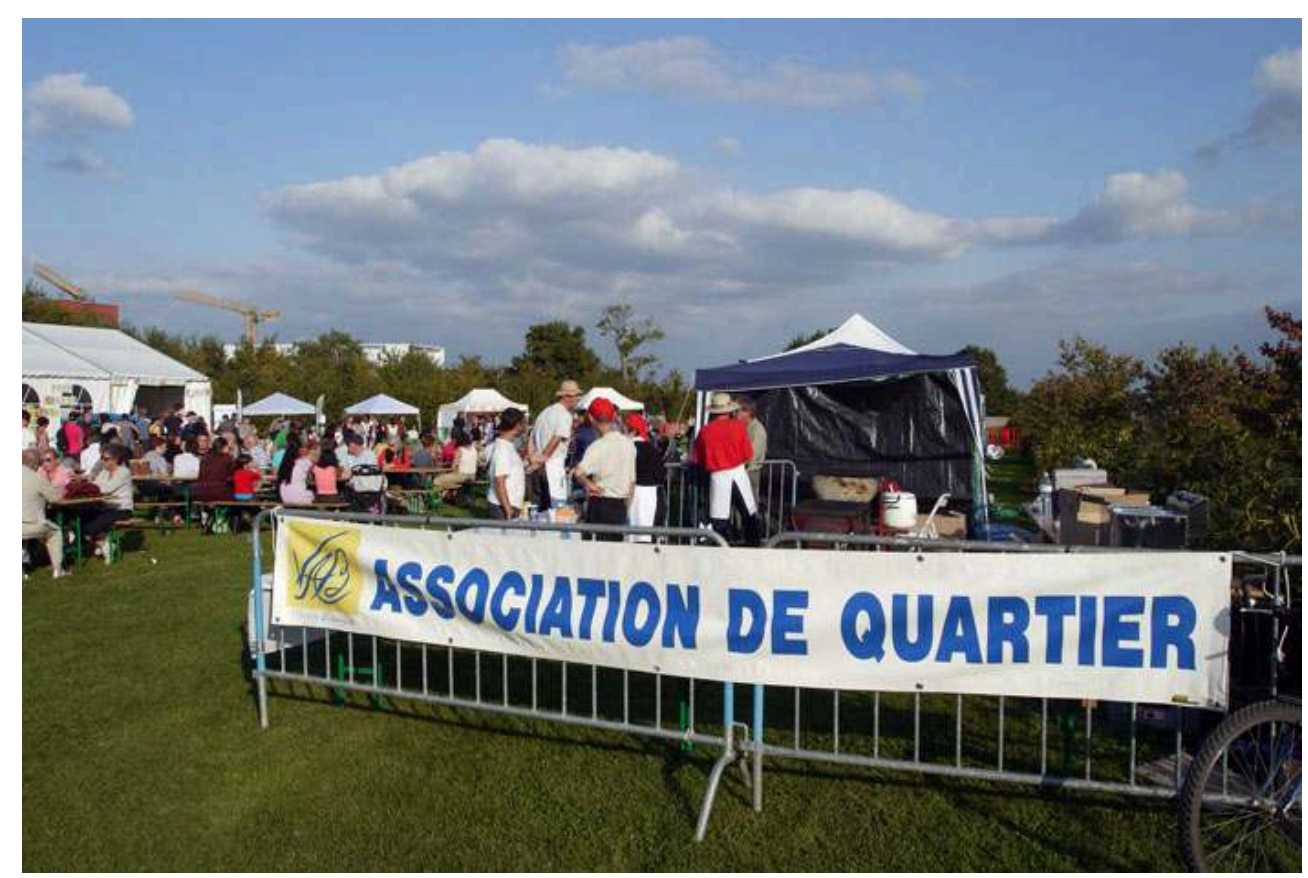

F. Valegeas, 2011

La nature des revendications et des actions portées par l'association, devenue un portevoix des attentes des habitants auprès des acteurs locaux, est révélatrice de la spécialisation des besoins exprimées, d'abord sur la question des enfants et sur le cadre de vie, puis plus généralement de la "vie de quartier » autour d'activités sportives et culturelles. L'action de l'association de quartier au sein des commissions de concertation mises en place par l'aménageur et la ville traduit ces sensibilités, en cherchant à préserver un cadre de vie jugé privilégié. 


\section{De l'attention au cadre de vie à l'engagement pour un « bien vivre ensemble »} par une nouvelle génération de bénévoles davantage sensibilisés à l'écologie, elle a cherché à articuler ses activités socio-culturelles avec sa commission interne « développement durable » créée en 2007. L'objectif est de redonner un nouveau souffle au projet associatif du quartier, de re-construire un projet associant les habitants, qu'ils soient anciens ou nouveaux arrivants, et les acteurs locaux. Il faut noter aussi que ces dernière années ont vu arriver au sein de l'association plusieurs membres qui nous ont signifié avoir un engagement fort pour l'écologie, par exemple pour plusieurs d'entre eux en étant engagés dans des partis écologistes ou des associations spécialisées. C'est à l'occasion de la refondation de son projet associatif en 2012 que Vivre à Beauregard a redéfini sa vocation: "association d'habitants pour un quartier durable ${ }^{24}$. Le terme quartier durable est ici employé non pas en référence aux modèles européens, mais redéfini localement comme un projet de vie collective basé sur une définition locale du « durable ». La présidente explique : « pour nous, un quartier durable c'est un quartier où les habitants se prennent en main et montent des projets ensemble. C'est pas parler de trucs compliqués comme le HQE [...] Nous parlons de "bien vivre ensemble ", c'est aller plus loin que de parler d'enfance, de sport ou d'aménagement, mais de penser ça comme un tout. On parle de projet de territoire [...], un projet de société à l'échelle du quartier ${ }^{25}$.

Vivre à Beauregard se réapproprie le développement durable comme support d'un projet de "bien vivre ensemble », c'est-à-dire comme projet local de vie collective qui permettrait le renforcement de cette communauté de destins autour d'une perspective partagée. Dans cette dynamique, l'association cherche à devenir un creuset pour les initiatives locales, en stimulant les alternatives, en devenant des lieux d'expérimentation et de construction de savoirs partagés. Ainsi, sous son impulsion ou avec son accompagnement, des liens ont été établis avec une association écologiste hébergée en bordure du quartier, afin de créer une Association pour le Maintien d'une Agriculture Paysanne baptisée Bioregard ${ }^{26}$. D'autre part, elle a développé des animations à visée plus pédagogique, que cela soit autour de l'art (ateliers land art), de la découverte du territoire (découverte de la faune et de la flore), ou à destination des enfants (projets menés en lien avec l'école du quartier). L'association cherche notamment à sensibiliser le plus largement les habitants à sa démarche, et a produit en 2013 un guide de restitution des paroles d'habitants recueillies au sein d'ateliers thématiques. 
Figure 4 : Brochure de restitution d'une collecte de paroles d'habitants

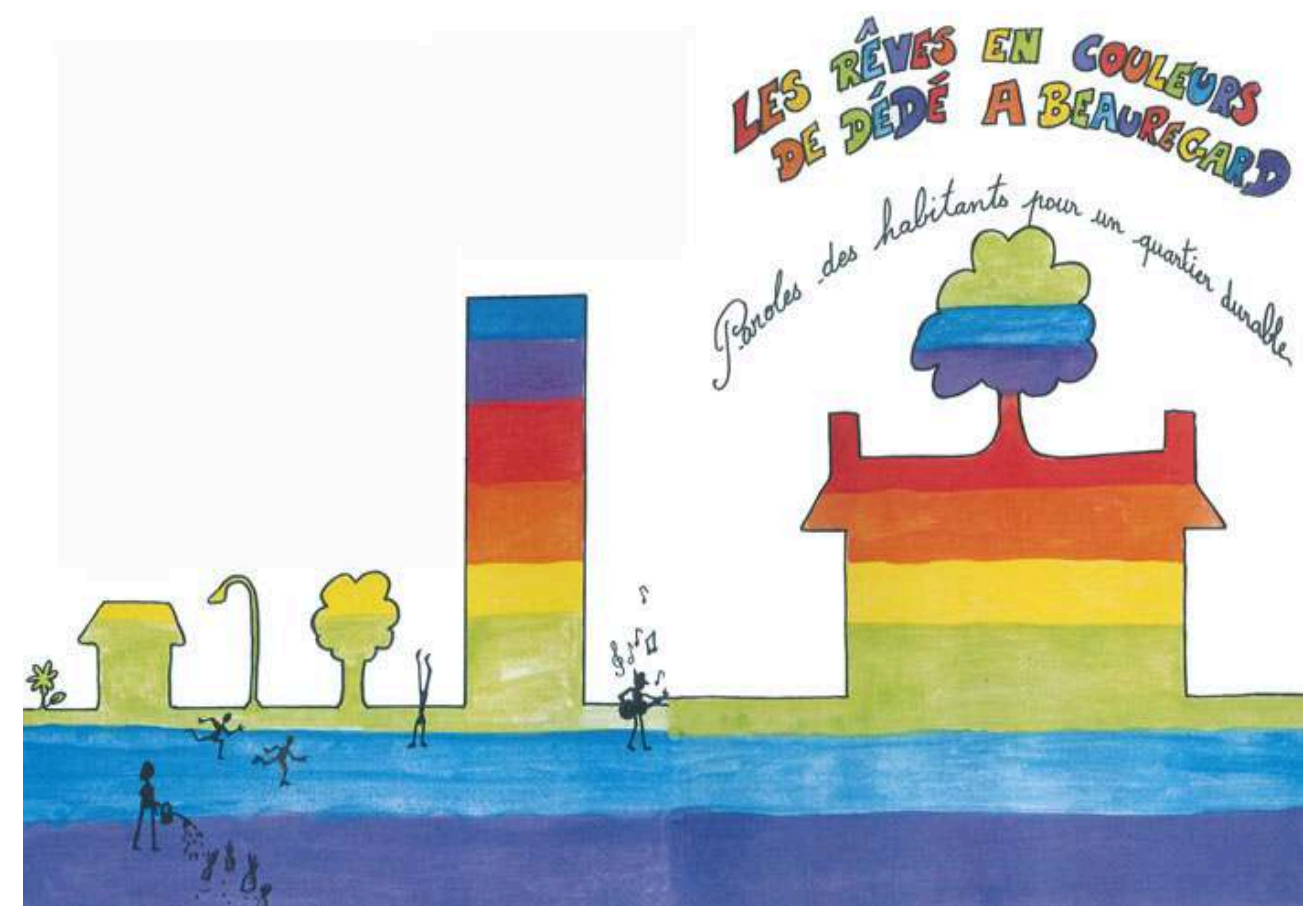

Vivre à Beauregard, 2013

Ce développement durable, principe fondateur du projet urbain mais qui paraît souvent flou aux habitants, est ainsi mobilisé comme point d'appui à un renouvellement du projet collectif du quartier. Cette construction d'un projet collectif se veut devenir aujourd'hui un creuset pour des expérimentations locales, qu'elles soient d'initiative habitantes, associatives, ou plus institutionnelles ${ }^{27}$. L'objectif est de s'appuyer sur un rapport sensible que les habitants entretiennent avec leur quartier, lié notamment à ses caractéristiques physiques et urbaines, pour le transformer en un engagement dans la vie collective locale. C'est, selon les animateurs de la commission développement durable, un moyen d'impliquer les habitants dans le changement des modes de vie qui est nécessaire, et «de montrer que le développement durable c'est aussi des petites choses $d u$ quotidien, c'est une manière de vivre ensemble, moins consumériste. En fait, c'est «moins de biens, plus de liens $» ! »$.

\section{Des quartiers dits durables entre contradictions et potentialités}

51 Ces dynamiques en cours dans l'exemple rennais marquent le passage d'une mobilisation autour d'enjeux environnementaux liés à l'aménagement du quartier vers un « projet collectif et politique » (Emelianoff, 2002), c'est-à-dire questionnant la place de l'intérêt collectif, du quartier dans la ville et plus largement des hommes dans leur environnement. Des auteurs ont ainsi montré que les formes de l'engagement, loin de s'estomper, avaient surtout évolué. La période actuelle est surtout marquée par une montée d'un "militantisme de proximité " (Ion, 2012), moins affilié. Les quartiers dits durables pourraient s'appuyer sur ces implications renouvelées dans la vie locale, sur des sensibilités accrues à l'écologie ou à des modes émergents de mutualisation (nouveaux modes de consommation, activités collectives ou coopératives), pour proposer la construction d'un devenir commun moins désincarné. Cette analyse 
souligne la nécessité de réfléchir aux conditions de l'appropriation des quartiers durables et aux potentialités de l'engagement des habitants. À rebours d'un individu rendu passif tel qu'il est bien souvent perçu et conçu dans les quartiers dits durables, des dynamiques alternatives peuvent émerger, basées sur une réappropriation des projets et des espaces. Elles peuvent alors constituer un support d'émancipation des individus et de réflexion pour un devenir commun. Ce serait une condition nécessaire au passage d'un "écoquartier dans l'espace", comme projet technique, à un "quartier durable dans le temps" (Boutaud, 2009), intégrant une réflexion sur les modes d'habiter individuels et collectifs. Le « durable » (ce qui dure) est alors interrogé et incite à mieux prendre en considération l'articulation entre d'une part les temps du projet, et d'autre part les temps sociaux de la construction d'un devenir. L'analyse des dynamiques à Beauregard montre l'importance de favoriser la construction d'espaces hétérotopiques ${ }^{29}$ permettant la construction d'un devenir commun: à la fois des espaces physiques favorisant l'appropriation et la construction d'identités, des espaces politiques laissant émerger et prendre corps les demandes sociales, et enfin des espaces temporels permettant la projection collective.

52 La construction de tels "collectifs " serait alors un second âge du développement durable (Theys et al., 2010), une « repolitisation par le bas » du développement durable en quelque sorte ${ }^{30}$.

\section{Conclusion}

Des questionnements demeurent sur la place de la diversité sociale dans la construction de telles initiatives collectives.

Tout d'abord, faut-il déduire de cet exemple que c'est l'homogénéité des trajectoires sociales et familiales qui permet l'émergence de ces formes d'engagement? Cela rejoindrait un discours justifiant une forme de spécialisation sociale de ces quartiers : « nous sommes tous dans des entre-soi familiaux, professionnels, amicaux. Au moins, dans ces quartiers, l'entre-soi met en route une expérimentation sociale très intéressante " (Emelianoff, 2008). Un important défi est là aujourd'hui : pérenniser une réflexion autour de ce projet collectif tout en développant des stratégies d'insertion des nouveaux habitants, aux profils plus diversifiés, ayant des trajectoires sociales et résidentielles ou des aspirations qui ne les amènent pas à s'inscrire naturellement dans cette communauté de destins. À ce propos, l'exemple de l'association Vivre à Beauregard est intéressant. En effet, les débats autour du développement durable ont été initiés par des adhérents appartenant souvent à des groupes sociaux traditionnellement davantage sensibles à l'écologie (diplômés du supérieur, urbains) ${ }^{31}$, voire même des militants de l'écologie. Mais l'association tient à sensibiliser le plus grand nombre par la variété des activités qu'elle propose, ou par l'organisation d'évènements tels que la fête de quartier. Mais, selon les mots de la présidente de Vivre à Beauregard, il s'agit d'un « chantier encore en cours $»^{32}$.

D'autre part, ces projets posent des questions quant à leur intégration dans la ville. Ils sont en effet suspectés de favoriser des mécanismes de ségrégation, des «entre-soi » fondés à la fois sur une capacité financière des ménages à s'installer dans des nouveaux quartiers valorisant une " haute qualité de vie », et partageant des valeurs et modes de vie semblables comme on a pu le voir dans l'analyse du cas rennais. Cette tendance à la spécialisation reste à démontrer dans la diversité des quartiers durables et dans une 
temporalité plus longue, au-delà de quelques constats élaborés à partir de cas spécifiques. Néanmoins, deux hypothèses se dessinent, dont il sera intéressant de comprendre les ressorts :

- d'une part un renfermement sur soi de cette « communauté de valeurs », cherchant à mettre en œuvre une exemplarité interne par la création d'ilots de durabilité, et rejetant les externalités négatives vers le reste de la ville;

- ou, à l'inverse, la prise de conscience d'un devenir commun par une articulation entre l'action locale et une ouverture vers la ville mettant ainsi en œuvre le « penser global, agir local » auquel se réfèrent les tenants d'une écologie politique.

\section{BIBLIOGRAPHY}

Ascher F., 2004, Les nouveaux principes de l'urbanisme, Paris, L’Aube, 110 p.

Authier J.-Y., Bacqué M.-H., Guerin-Pace F. (dir), 2006, Le quartier. Enjeux scientifiques, actions politiques et pratiques sociales, Paris, La Découverte, $293 \mathrm{p}$.

Baudin G., Dupuy S., 2001, « Le village ambigu. Des voisins dans la ville », in P.U.C.A., Les annales de la recherche urbaine, dossier « les seuils du proche », $\mathrm{n}^{\circ} 90, \mathrm{p} .77-84$

Béal V., Gauthier M., Pinson G., 2011, Le développement durable changera-t-il la ville ? Le regard des sciences sociales, Saint-Etienne, Publications de l'Université de Saint-Etienne, $461 \mathrm{p}$.

Bonard Y., Matthey L., 2009, « Les éco-quartiers : laboratoires de la ville durable. Changement de paradigme ou éternel retour du même ? ", Cybergeo : European Journal of Geography (en ligne), mis en ligne le 9 juillet 2010, consulté le 8 mars 2011, URL : http://cybergeo.revues.org/23202

Boissonnade J., 2011, « Le développement durable face à ses épreuves », in Levy A., Emelianoff C., «Quelle ville durable?», Espaces et sociétés, n¹47, p.57-75

Boutaud B., 2009, « Quartier durable ou éco-quartier? ", Cybergeo : European Journal of Geography (en ligne), mis en ligne le 24 septembre 2009, consulté le 8 mars 2011, URL : http://

cybergeo.revues.org/22583

Conseil d'analyse stratégique, 2011, « « Nudges verts » : de nouvelles incitations pour des comportements écologiques ", note d'analyse, $12 \mathrm{p}$.

Emelianoff C., 2002, "Comment définir une ville durable », mis en ligne le 13 juin 2002, consulté le 18 octobre 2011, URL : www.ecologie.gouv.fr/IMG/ agenda21/intro/emelia.htm

Emelianoff C., 2008, « Entretien », La revue durable, Fribourg, p.27-29

Giband D., 2011, Les villes de la diversité. Territoires du vivre ensemble, Paris, Economica, Anthropos, $150 \mathrm{p}$.

Hamman P., 2012, Sociologie urbaine et développement durable, Bruxelles, De Boeck, 199 p.

Ion J., 2012, S'engager dans une société d'individus, Paris, Armand Colin, 214 p.

Kokoreff M., Lapeyronnie D., 2013, Refaire la cité. L'avenir des banlieues, La République des idées, Paris, Seuil, 107 p. 
Lascoumes P., 2001, «Les ambiguïtés politiques du développement durable », Université de tous les savoirs, La nature et les risques, Vol. 6, Paris, Odile Jacob, p.250-263

Ledrut R., 1968, Sociologie urbaine, Paris, Presses Universitaires de France, 224 p.

Lefebvre H., 1967, «Quartieret vie dequartier »,Cahiers de l'IAURP, $n^{\circ} 7$.

Lefebvre H., 1974, La production de l'espace, Paris : Anthropos, 485 p.

Lelevrier C., 2006, Les mixités sociales, Problèmes politiques et sociaux, Paris, La documentation française, $\mathrm{n}^{\circ} 929,120 \mathrm{p}$.

Ministère de l'Ecologie, du Développement Durable, des Transports et du Logement, 2008, Notice explicative du dossier de candidature au concours EcoQuartier 2008-2009, 23 p.

Ministère de l'Ecologie, du Développement Durable, des Transports et du Logement, 2011, Appel à projets EcoQuartier, notice explicative de la grille EcoQuartier 2011, 31 p.

Morin R., Rochefort M., 1998, «Quartier et lien social : des pratiques individuelles à l'action collective », Lien social et Politiques, n³9, p.103-114.

P.U.C.A., 2006, « Programme Quartiers durables », Dossier Premier Plan, nº12.

Raineau L., 2009, « Deux expériences comparées d'écoquartiers. Bedzed à Londres et Vauban à Fribourg ", in Dobre M., Juan S., Consommer autrement. La réforme écologique des modes de vie, Paris, L'Harmattan, p.73-84.

Rautenberg M., Lefebvre B., 2010, Utopies et mythologies urbaines à Villeneuve d'Ascq, Villeneuve d'Ascq, Presses Universitaires du Septentrion, 224 p.

Renauld V., 2012, Fabrication et usage des écoquartiers français, thèse de doctorat, Institut National des Sciences Appliquées de Lyon.

Souami T., 2009, Ecoquartiers : secrets de fabrication. Analyse critique d'exemples européens, Paris, Les carnets de l'info, $252 \mathrm{p}$.

Theys J., 2002, «L'approche territoriale du « développement durable », condition d'une prise en compte de sa dimension sociale », Développement durable et territoires (en ligne), mis en ligne le 23 septembre 2002, consulté le 9 mars 2011, URL : http://developpementdurable.revues.org/1475

Theys J., du Tertre C., Rauschmayer F., 2010, Le développement durable, la seconde étape, Paris, L'aube, $205 \mathrm{p}$.

Ville de Rennes, 2007, Pour un aménagement durable à Rennes. Actes du séminaire des urbanistes du 5 juillet 2007, Rennes, $121 \mathrm{p}$.

Zetlaoui-Léger J. (dir), 2013, La concertation citoyenne dans les projets d'éco-quartiers en France : évaluation constructive et mise en perspective européenne, rapport de recherche du programme Concertation Décision Environnement, 270 p.

\section{NOTES}

1. Nous nommerons "quartiers dits durables" tous les quartiers se réclamant de l'urbanisme durable, qu'ils soient qualifiés « éco-quartiers », « quartiers durables »...

2. Par exemple la "ville libre de Christiana» à Copenhague, ou le mouvement Vauban à Fribourg-en-Brisgau.

3. Engagement Grenelle, table ronde finale du Grenelle Environnement, octobre 2007. 
4. Ces appels à projets ont rencontré un succès inattendu (plus de 160 candidats en 2009, 360 en 2011).

5. Entretien avec le chef de projet Écoquartiers au ministère, le 8 avril 2011.

6. Préface de Guy Di Meo, in Giband, 2011, p.8.

7. Renvoyant à la politique définie par J. Rancière (Aux abords du politique, 2003)

8. Certains acteurs notamment nationaux citent aujourd'hui couramment Beauregard comme l'un des précurseurs des quartiers durables, reconstruisant ainsi a posteriori l'histoire de sa conception et de son inscription dans un développement urbain durable.

9. Entretien avec le responsable d'opération du projet de Beauregard, le 22 avril 2011.

10. Un élu de quartier, sur son blog, le 9 janvier 2011 (consulté le 10 mars 2012).

11. Entretien avec le responsable d'opération du projet de Beauregard, le 22 avril 2011.

12. Un élu de quartier, sur son blog, le 9 janvier 2011.

13. Entretien avec le responsable d'opération du projet de Beauregard, le 22 avril 2011.

14. Propos du responsable d'opération du projet de Beauregard, lors d'un parcours commenté, le 25 mai 2012.

15. Entretien avec un membre de Vivre à Beauregard, le 23 septembre 2011.

16. Une femme retraitée de 68 ans, habitante du quartier depuis 9 ans.

17. Un couple de fonctionnaires, 36 et 39 ans, habitants du quartier depuis 6 ans.

18. Ouest France, le 22 octobre 2003.

19. Un cadre de 44 ans, habitant du quartier depuis 12 ans.

20. A titre d'exemple, en $2006,50,6 \%$ de la population a entre 20 et 39 ans, contre $38,8 \%$ sur le reste de la commune; de même les CSP+ représentent $35,8 \%$ de la population contre $28,3 \%$ sur l'ensemble de la commune (chiffres APRAS).

21. Entretien avec un élu de Rennes Métropole, le 21 février 2012.

22. Un fonctionnaire de 31 ans, engagé au sein d'autres associations dans la ville, notamment écologistes, entretien du 28 novembre 2011.

23. Cette mobilisation a pris la forme d'une pétition et d'une étude des besoins réalisée par les habitants dès 2002.

24. Nouveau slogan de l'association formulé au printemps 2012.

25. Entretien du 18 mars 2013.

26. L'AMAP rassemble une trentaine d'adhérents depuis sa création en juillet 2013.

27. Habitat intergénérationnel innovant, maison de quartier dont le programme est co-élaboré dans une démarche participative, réaménagement d'une zone humide à l'initiative de l'association de quartier...

28. Entretien du 19 mars 2013.

29. Au sens de Lefebvre (1974) : lieux d'expression des particularismes, de la construction des attachements et des appropriations individuelles et collectives, à l'inverse des isotopies, espaces forgés par la technique et les normes sociales.

30. Dans l'esprit de ce que développent M. Kokoreff et D. Lapeyronnie (2013) dans un tout autre contexte

31. Lire à ce sujet MEDDTL, 2011, « Les perceptions sociales et pratiques environnementales des Français de 1995 à 2011 ", La revue du CGDD, 68 p.

32. Entretien du 18 mars 2013. 


\section{ABSTRACTS}

The number of sustainable districts has been growing in France for the last few years. These districts, though aiming to offer a "new art of living together", often provide only technical and normative answers to sustainable development issues. For example, dealing with social issues is done through the point of view of diversity of housing without a real renewing of this approach. Inhabitants are only considered through their acceptation of the projects, their behavior and if their lifestyles are sustainable or not. There is not much said about imagining new individual or collective ways of living in these neighborhoods. The results of an empirical research question possible forms of appropriation and allow the emergence of collective dynamics. The example of Beauregard district in Rennes shows how inhabitants can take up on the issue of a sustainable development, redefining and re-appropriating it as a base for the construction of a collective future.

Les projets de quartiers dits durables se multiplient en France depuis quelques années. Mais ces quartiers, s'ils affichent vouloir créer un "nouvel art de vivre ensemble ", n'apportent bien souvent que des réponses techniques et normatives aux enjeux du développement durable. Les «dimensions sociales », sont traitées d'abord sous l'angle d'une diversité de l'habitat, sans réel renouvellement de cette approche. Les habitants sont perçus à l'aune de leur acceptation des projets, et de leurs comportements, durables ou non. Peu de choses sont dites sur le renouvellement des modes d'habiter individuels ou collectifs dans ces quartiers. Les résultats d'une recherche empirique interrogent les formes d'appropriations possibles, permettant l'émergence de dynamiques collectives. L'exemple du quartier de Beauregard à Rennes questionne la manière dont les habitants peuvent se saisir d'un développement durable, le redéfinir et se le réapproprier comme support pour la construction d'un devenir commun à l'échelle du quartier.

Die Projekten von sogenannten nachhaltigen Stadtvierteln vermehren sich in Frankreich seit einigen Jahren. Der von diesen Vierteln angestrebte Wunsch lautet, ein neues Modell des Zusammenlebens zu schaffen. Sie liefern aber oft nur technische und normative Antworten auf die Herausforderungen der nachhaltigen Entwicklung. Die sozialen Dimensionen werden vorrangig im Zusammenhang mit der Vielfalt der Wohnformen behandeln und es gibt keine neue Herangehensweise an diese Frage. Was den Bewohnern angeht, wird vor allem in Betracht gezogen, ob sie die Projekten annehmen und nachhaltig verhalten. Wenig wird über die Erneuerung der Wohnformen in diesen Vierteln geschrieben. Angesichts der Ergebnisse einer empirischen Studie stellt sich die Frage nach den Aneignungsformen, die die Entstehung einer kollektiven Dynamik ermöglichen. Mit dem Beispiel des «Beauregard » Viertels in Rennes, wird hinterfragt, wie sich die Bewohner der nachhaltigen Entwicklung aneignen und sie als Stütze für den Aufbau eines Gemeinsam-Werdens neu bestimmen können.

\section{INDEX}

Schlüsselwörter. Beteiligung, Bewohner, Gemeindeprojekte, nachhaltiges Stadtviertel, Zusammenleben

Mots-clés: écoquartiers, habitants, participation, projets collectifs, quartiers durables, vivre ensemble

Keywords: collective projects, eco-districts, inhabitants, living together, participation, sustainable neighborhoods 


\section{AUTHOR}

\section{FRANÇOIS VALEGEAS}

Doctorant au Laboratoire Ville Mobilité Transports / Lab'Urba, Université Paris-Est - Institut d'urbanisme de Paris, 61 Avenue du Général de Gaulle, 94010 Créteil Cedex -

francoisvalegeas@hotmail.fr 